\title{
EINFÜHRUNG \\ IN DIE MATHEMATISCHE LOGIK
}

\author{
TE I L I I \\ PRÄDIKATENKALKÜL DER ERSTEN STUFE \\ Von Dr. sc. nat. G ̈̈N TER ASSER \\ o. Professor für Mathematische Logik und Grundlagen der Mathematik \\ an der Sektion Mathematik der Ernst-Moritz-Arndt-Universität Greifswald
}

2. Auflage

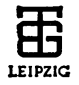

BSB B.G.TEUBNER VERLAGSGESELLSCHAFT

$$
1975
$$


ISBN 978-3-322-00718-6 ISBN 978-3-322-91274-9 (eBook)

DOI $10.1007 / 978-3-322-91274-9$

$$
\text { VLN } 294-375 / 43 / 75 \quad \text { LSV } 1094
$$

(C) BSB B.G.Teubner Verlagsgesellschaft, Leipzig, 1972

Lektor: Dorothea Ziegler

Satz: GG Interdruck Leipzig

Bestell-Nr. 6656312

EVP 14,50 Mark 


\section{VORWOR'T}

Nachdem seit dem Erscheinen des ersten Teiles der „Einführung in die Mathematische Logik" mehr als 12 Jahre vergangen sind, bin ich nunmehr in der Lage, den zweiten Teil vorzulegen. Entsprechend dem ursprünglichen Plan enthält er die wichtigsten Ergebnisse über die Prädikatènlogik der ersten Stufe, die hier konsequent als Logik (einsortiger) elementarer Sprachen entwickelt wird. Ich behandle dabei sofort den Prädikatenkalkül mit Identität und Operationssymbolen; die zum Teil abweichenden Ergebnisse für den Prädikatenkalkül ohne Identität erscheinen als Resultate über Ausdrücke und Ausdrucksmengen, in denen das Gleichheitszeichen nicht vorkommt. Das hat den Vorteil, daß die Prädikatenlogik der ersten Stufe sofort in einer solchen Allgemeinheit aufgebaut wird, wie man sie in der mathematischen Grundlagenforschung (Metamathematik) beim Studium formalisierter elementarer Theorien in der Regel auch tatsächlich benötigt.

Wie auch im ersten Teil, geht es mir vor allem um die Darstellung der Wechselbeziehungen zwischen semantischen und syntaktischen Fragestellungen. Dabei habe ich mich insbesondere darum bemüht, die Rolle des Modellbegriffs und des auf ihm basierenden Begriffs des logischen Folgerns deutlich herauszuarbeiten. Ich halte den Folgerungsbegriff für den eigentlichen zentralen Begriff der Logik, dessen formale (syntaktische) Erfassung das Hauptproblem der mathematischen Logik darstellt. Dabei kommt dieser formalen Erfassung in der Prädikatenlogik wegen der Nichtentscheidbarkeit der Folgerungsrelation, die allerdings erst im dritten Teil bewiesen wird, grundsätzliche Bedeutung zu.

Die in dieser „Einführung“ vertretene semantische Grundauffassung besteht, wie bereits im Vorwort zum ersten Teil angedeutet, in der Anerkennung einer (etwa durch Verwendung von Klassen) von den bekannten Antinomien gereinigten naiven Mengenlehre mit Auswahlaxiom. Neben dem Mengenbegriff werden insbesondere der Abbildungsbegriff und die auf diesem beruhenden Begriffe Kardinal- und Ordinalzahl als inhaltlich gesicherte mathematische Begriffe anerkannt. In einem Anhang bringe ich die wichtigsten Fakten aus der allgemeinen Mengenlehre, insbesondere über Kardinal- und Ordinalzahlen, die über das heute übliche mengentheoretische Allgemeinwissen hinausgehen und die mir für das richtige Verständnis einer Reihe von Begriffen, Sätzen und Beweisen 
notwendig erseheinen. Der Leser, der mit den einfachsten Grundbegriffen über Kardinal- und Ordinalzahlen nicht vertraut ist, kann diesen Anhang entweder vor dem eigentlichen Studium des Prädikatenkalküls oder im Bedarfsfall studieren.

Die gewählte Darstellungsweise ist in dem Sinne als ,kklassisch“ zu bezeichnen, als alle Betrachtungen im wesentlichen in der Allgemeinheit und in den Begriffen durchgeführt werden, in denen die eigentlichen Probleme formuliert sind. Es wird also bewußt darauf verzichtet, die Probleme in einen (derzeit) allgemeinsten Zusammenhang einzuordnen und sie als Spezialfälle von wesentlich allgemeineren Fragestellungen nachzuweisen. In den letzten Jahren wurden auch in der mathematischen Logik in dieser Hinsicht viele bemerkenswerte Resultate erzielt, insbesondere wurden grundlegende Zusammenhänge mit der Theorie spezieller Arten von Booleschen Algebren entdeckt. Ich bin jedoch der Meinung, daß die Herausarbeitung dieser allgemeinen Zusammenhänge nicht Gegenstand einer Einführung in die mathematische Logik sein kann, zumal hierbei leicht die Gefahr besteht, daß das eigentliche inhaltliche Anliegen im Gestrüpp abstrakter Begriffe und Methoden verloren geht.

Ich möchte zum Abschluß meinen Mitarbeitern Herrn Dr. P. ScHREIBER und Herrn Dr. H.-D. Hecker für die Hilfe danken, die sie mir beim Lesen der Korrekturen gewährt haben. Mein Dank gilt ferner dem Verlag, der auch bei diesem zweiten Teil meine Wünsche bezüglich der Gestaltung des Bandes in großzügiger Weise erfüllte, sowie den Setzern des GG Interdruck Leipzig, die sich redlich bemüht haben, den schwierigen Formelsatz zu meistern. 


\section{N H A LT}

$\S$ 1. Attribute und Quantifizierungsfunktionen $\ldots \ldots \ldots \ldots \ldots \ldots \ldots \ldots$. 1

$\S 2$. Ausdrücke einer elementaren Sprache $\ldots \ldots \ldots \ldots \ldots \ldots \ldots \ldots \ldots$

$\S 3$. Interpretation der Ausdrücke einer elementaren Sprache . . . . . . . 22

$\S$ 4. Reduktions- und Repräsentantentheoreme .............. 50

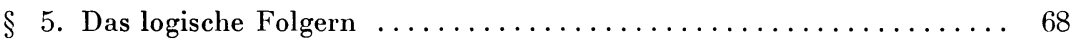

$\S 6$. Ableitbarkeit und Beweisbarkeit $\ldots \ldots \ldots \ldots \ldots \ldots \ldots \ldots \ldots$

$\S$ 7. Grundlegende Gesetze der Prädikatenlogik................ 89

$\S$ 8. Die Axiomatisierungstheoreme für den Prädikatenkalkül der ersten

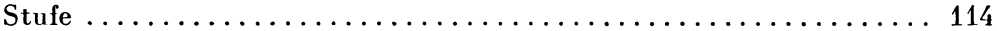

$\S$ 9. Prädikatenlogische Normalformen .................. 135

$\S 10$. Der bestimmte Artikel $\ldots \ldots \ldots \ldots \ldots \ldots \ldots \ldots \ldots \ldots \ldots \ldots \ldots \ldots$

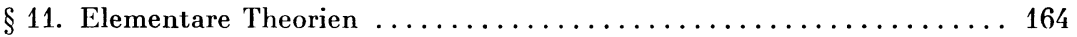

Anhang - Einiges aus der allgemeinen Mengenlehre ........... 170

Literaturverzeichnis $\ldots \ldots \ldots \ldots \ldots \ldots \ldots \ldots \ldots \ldots \ldots \ldots \ldots \ldots$

Namen- und Sachverzeichnis........................ 184 